Supplement of The Cryosphere, 13, 2087-2110, 2019

https://doi.org/10.5194/tc-13-2087-2019-supplement

(C) Author(s) 2019. This work is distributed under

the Creative Commons Attribution 4.0 License.

(c) (1)

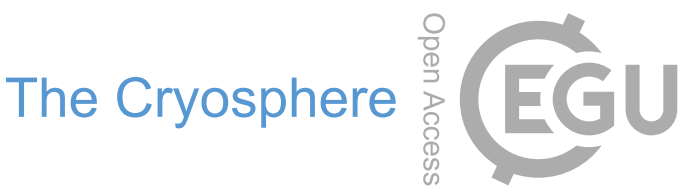

Supplement of

\title{
Permafrost variability over the Northern Hemisphere based on the MERRA-2 reanalysis
}

\section{Jing Tao et al.}

Correspondence to: Jing Tao (jingtao@lbl.gov)

The copyright of individual parts of the supplement might differ from the CC BY 4.0 License. 
Uncertainty of the Sampling Mean. Statistically, the measurement uncertainty over a sampling area is the uncertainty of the average at the related scale. That is, the uncertainty of the ALT average (over the sampling area) is the standard deviation normalized by the square root of sampling size according to the Central Limit Theorem (CLT). Specifically, given M measurements of ALT within a certain sampling area, one can randomly draw K measurements to compute an averaged ALT (ALT avg); by repeating such random sampling procedure by $\mathrm{N}$ times, one will have $\mathrm{N}$ independent $\mathrm{ALT}_{\text {avg }}$ values. If $\mathrm{K}$ and $\mathrm{N}$ both are sufficiently large, those $\mathrm{N}$ independent $\mathrm{ALT}_{\text {avg }}$ values will form a normal distribution with a mean close to the mean of all measurements, and a standard deviation related to the sampling size $K$, i.e., sigma/sqrt(K) where sigma is the standard deviation of the $\mathrm{M}$ measurements. For example, given a spatial standard deviation of $\sim 0.1 \mathrm{~m}$ within CALM sites at the $100 \mathrm{~m} \times 100 \mathrm{~m}$ scale and the fact that $\sim 100$ measurements are available at each such site, the uncertainty estimate of the average is about 0.01 $\mathrm{m}(=0.1 / \mathrm{sqrt}(100))$.

Uncertainty Propagation. The measurement uncertainty is tightly associated with the sampling area and thus is scale-dependent. To estimate the total uncertainty propagated from small to large scales, we employed the method of Schaefer et al. (2015) by adding in quadrature the uncertainty components from each involved scale/level.

Uncertainty of AirMOSS retrievals. For AirMOSS retrievals, the overall uncertainty is the quadrature sum of two-level uncertainties, i.e., the retrieving uncertainty at AirMOSS native resolution $(20 \mathrm{~m} \times 60 \mathrm{~m})$ and the uncertainty of mean ALT retrieval over the model grid cell (i.e., the standard deviation of ALT retrievals normalized by the sampling size). Due to the small spatial variability and large sample size (i.e., considering the number of pixels at $20 \mathrm{~m} \times 60 \mathrm{~m}$ resolution 
within an $81-\mathrm{km}^{2}$ model grid cell) of retrievals, the uncertainty of the ALT mean aggregated to the model grid-scale is very small. Thus, the retrieving uncertainty dominates the overall uncertainty in the ALT retrievals.

Uncertainty for CALM in-situ Measurements. When comparing in-situ measurements with simulation results at the model grid-scale, the overall uncertainty for in-situ measurements should include the uncertainties from three levels, i.e., the probing uncertainty, the uncertainty of mean ALT at the CALM site scale, and the upscaling errors from the site scale to the model scale, as illustrated by the Figure S1.

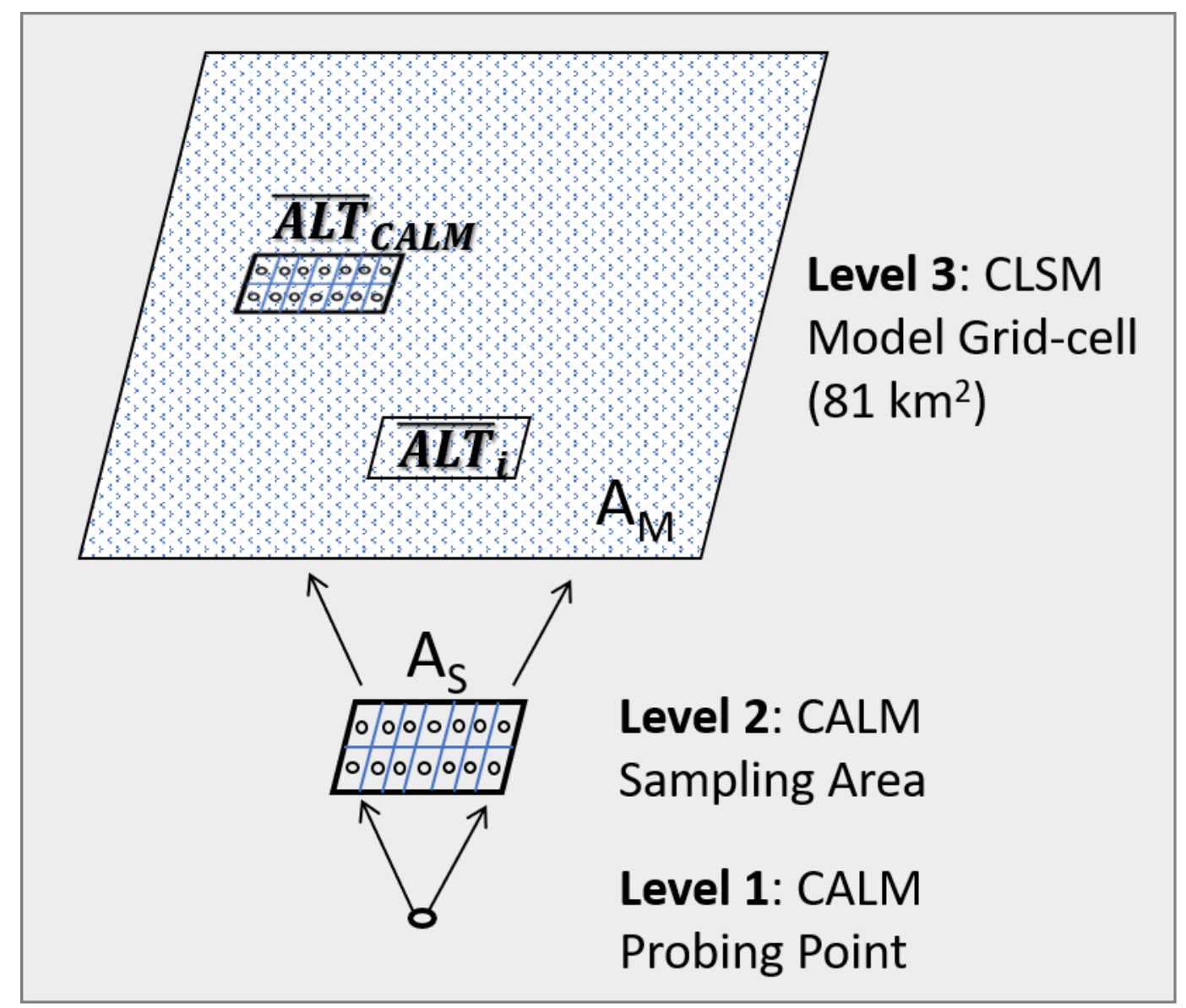

Figure S1: ALT measurements at a variety of scales. The measurement uncertainties propagate from a probing point to a model grid-cell area of $81 \mathrm{~km}^{2}$. 
A general strategy to estimate the total uncertainty $(\sigma)$ is to add in quadrature the uncertainty from each level (three levels in this case), as expressed in the equation below.

$$
\sigma=\sqrt{\sigma_{3}^{2}+\sigma_{2}^{2}+\sigma_{1}^{2}}
$$

where $\sigma_{1}$ represents the level-1 probing uncertainty, $\sigma_{2}$ is the uncertainty of the mean ALT at the level-2 (or site-) scale, calculated as the standard deviation of measurements over the sampling area (As) normalized by the sampling size, and $\sigma_{3}$ represents the level-3 upscaling error from sitescale (As) to model grid-cell scale (Aм), similarly expressed as the standard deviation of the averaged ALTs $\left(\overline{A L T}_{i}\right)$ of the same area (As) of the CALM sampling patch all over the model grid cell normalized by the sampling size (note the population size is approximately $\mathrm{A}_{\mathrm{M}} / \mathrm{As}_{\mathrm{s}}$ ). In reality, it is impossible to obtain this upscaling error, and thus the overall uncertainty for the measurements at the model grid scale cannot be realistically estimated.

Representative Error for the Point-To-Grid Comparison. We then disregard the statistical uncertainty for in-situ measurements at the model scale. Instead, we show the spatial variability of the measurements at the site scale to illustrate how heterogeneous the sampling area is in terms of ALT and thus how the site-scale sampling area is representative for a larger scale. This uncertaintyto-variability displacement was also adopted in other studies (Chen et al., 2019; Schaefer et al., 2015). Such displacement between statistical uncertainty (expressed as the standard deviation normalized by the square root of sampling size) and spatial variability (expressed as the standard deviation) is particularly helpful for interpreting the spatial representative error of in-situ measurements associated with the point-to-grid comparison. However, one should notice that this estimate (simply the standard deviation) here is far away from the statistically "true" uncertainty of the average at the model grid-cell scale. 


\section{Reference}

Chen, R. H., Tabatabaeenejad, A., and Moghaddam, M.: Retrieval of permafrost active layer properties using time-series P-band radar observations, IEEE Transactions on Geoscience and Remote Sensing, 10.1109/TGRS.2019.2903935, 2019.

Schaefer, K., Liu, L., Parsekian, A., Jafarov, E., Chen, A., Zhang, T. J., Gusmeroli, A., Panda, S., Zebker, H. A., and Schaefer, T.: Remotely Sensed Active Layer Thickness (ReSALT) at Barrow, Alaska Using Interferometric Synthetic Aperture Radar, Remote Sens-Basel, 7, 3735-3759, 2015. 